MATHEMATICS OF COMPUTATION

Volume 67, Number 224, October 1998, Pages 1383-1400

S 0025-5718(98)00970-3

\title{
VORTEX METHOD FOR TWO DIMENSIONAL EULER EQUATIONS IN BOUNDED DOMAINS WITH BOUNDARY CORRECTION
}

\author{
LUNG-AN YING
}

\begin{abstract}
The vortex method for the initial-boundary value problems of the Euler equations for incompressible flow is studied. A boundary correction technique is introduced to generate second order accuracy. Convergence and error estimates are proved.
\end{abstract}

\section{INTRODUCTION}

The vortex methods are employed in the numerical simulation of incompressible flow. For viscous flow the Navier-Stokes equations are approximated by using an operator splitting between the inviscid Euler equations and the Stokes equations. One difficulty of the vortex methods is the treatment of boundary conditions. In the viscous step a vorticity creation operator is applied to maintain the no-slip condition. In the inviscid step the particle method is used to simulate the vorticity. The scheme is required to be consistent and stable. The purpose of this paper is to study this problem in the presence of boundaries. We restrict ourselves to the Euler equations only, because we think it is a necessary first step toward studying the problem for the entire scheme.

The consistency, stability and convergence of vortex methods for the initial value problems of the Euler equations was first obtained by Hald and DelPrete [9], [10], [11]; then the results were improved and different proofs were given by Anderson and Greengard [4], Beale and Majda [5], [6], and Raviart [13]. However there are some essential difficulties in the study of the vortex method for initial-boundary value problems. The high order consistency result depends on an application of Taylor's expansion which yields a superconvergence estimation. Therefore a uniform mesh is required for initial value problems, and it is difficult to get the same high order consistency for a bounded domain. Chiu and Nicolaides suggested some nonuniform meshes and introduced the derivatives of Dirac functions in the formula to get the necessary accuracy; however, the convergence result was proved for initial value problems.

To overcome the difficulty, the authors of [15] and [17] applied an extrapolation technique to make correction on the boundary which also gave high order convergence results for two dimensional initial-boundary value problems. Convergence of vortex methods for three dimensional initial-boundary value problems of the Euler

Received by the editor March 22, 1996 and, in revised form, April 23, 1997.

1991 Mathematics Subject Classification. Primary 65M99; Secondary 35Q35, 76C05.

Key words and phrases. Vortex method, Euler equation, initial boundary value problem.

(C)1998 American Mathematical Society 
equations was proved in [16], where for simplicity the initial data was assumed compactly supported. For general initial data the same boundary correction technique is also needed.

There are boundary layers for viscous flows. The gradient of the velocity field near the boundary is large, so the extrapolation approach causes a large error. We introduce a simpler boundary correction technique in this paper, one for which extrapolation is no longer needed. We will prove that this scheme possesses second order accuracy.

In the next section we will state our scheme. In $\S 3$ we will prove our convergence results. We will apply some results of the theory for initial value problems which can be found in the paper of Raviart [13], or in the book by the author and Zhang [18]. Finally we will give a numerical example which shows by contrast the difference among different treatment of boundary conditions.

\section{SCHEME}

For simplicity we assume that $\Omega$ is a bounded and convex domain in $\mathbb{R}^{2}$, the boundary of which, $\partial \Omega$, is sufficiently smooth. Let $x=\left(x_{1}, x_{2}\right)$ be the points in $\mathbb{R}^{2}$. We consider the following initial-boundary value problems:

$$
\begin{gathered}
\frac{\partial u}{\partial t}+(u \cdot \nabla) u+\frac{1}{\rho} \nabla p=0, \\
\nabla \cdot u=0, \\
\left.u \cdot n\right|_{\partial \Omega}=0, \\
\left.u\right|_{t=0}=u_{0},
\end{gathered}
$$

where $u=\left(u_{1}, u_{2}\right)$ is velocity, $p$ is pressure, $\rho$ is a constant density, $\nabla=\left(\partial_{1}, \partial_{2}\right)$, and $n$ is the outward unit normal vector on the boundary. Let $\omega=-\nabla \wedge u$ be vorticity, where $\nabla \wedge=\left(\partial_{2},-\partial_{1}\right)$, and $\psi$ be the stream function such that $u=\nabla \wedge \psi$. Then the vorticity-stream function formulation for the problem is

$$
\begin{gathered}
\frac{\partial \omega}{\partial t}+u \cdot \nabla \omega=0, \\
-\triangle \psi=\omega,\left.\quad \psi\right|_{\partial \Omega}=0, \quad u=\nabla \wedge \psi, \\
\left.\omega\right|_{t=0}=\omega_{0}=-\nabla \wedge u_{0} .
\end{gathered}
$$

If $u_{0}$ satisfies the consistency condition $\left.u_{0} \cdot n\right|_{\partial \Omega}=0$, and if it is sufficiently smooth, then there is a unique smooth solution on $\bar{\Omega} \times[0, T]$ for any $T>0$ [14].

Let $u^{\varepsilon}, \omega^{\varepsilon}, \psi^{\varepsilon}$ be the approximate velocity, vorticity, and stream function respectively, and $X_{j}^{\varepsilon}$ be the approximate characteristic curve, where $\varepsilon>0$ is a parameter and $j$ is an index corresponding to a particular particle. For each $j, X_{j}^{\varepsilon}$ satifies

$$
\frac{d X_{j}^{\varepsilon}}{d t}=u^{\varepsilon}\left(X_{j}^{\varepsilon}(t), t\right), \quad X_{j}^{\varepsilon}(0)=X_{j} .
$$

For simplicity we assume that the support of "vortex blob function" $\zeta$ is in the unit disk, supp $\zeta \subset S(o, 1)$; then we define $\zeta_{\varepsilon}(x)=\frac{1}{\varepsilon^{2}} \zeta\left(\frac{x}{\varepsilon}\right)$. As usual, we require that

$$
\int \zeta(x) d x=1 \text {. }
$$


For a given time $t, \omega^{\varepsilon}$ is a linear combination,

$$
\omega^{\varepsilon}(x, t)=\sum_{j \in J \cup J(t)} \alpha_{j}^{\varepsilon} \zeta_{\varepsilon}\left(x-X_{j}^{\varepsilon}(t)\right),
$$

where $J=\left\{j ; X_{j} \in \bar{\Omega}\right\}$, and $J(t)$ is the set of additional particles. Let us explain it in detail.

For each particle near the boundary we fix one reference point, $X_{j_{0}}^{\varepsilon}$, on the boundary at $t=0$. For definiteness we may take $X_{j_{0}}^{\varepsilon}$ to be the nearest point to $X_{j}$. According to (8) we get $X_{j_{0}}^{\varepsilon}$ for all $t$. We define

$$
X_{j^{\prime}}^{\varepsilon}(t)=2 X_{j_{0}}^{\varepsilon}(t)-X_{j}^{\varepsilon}(t), \quad \alpha_{j^{\prime}}^{\varepsilon}=\alpha_{j}^{\varepsilon},
$$

which yields one term in (9) with $j^{\prime} \in J(t)$. Because the equation (5) is homogeneous, $\alpha_{j}^{\varepsilon}$ does not change with time. If the distance from $X_{j^{\prime}}^{\varepsilon}$ to $\partial \Omega$ is greater than $\varepsilon$, then this term has no contribution, so only those $X_{j}^{\varepsilon}$ in a neighborhood of $\partial \Omega$ need to be taken into account.

The equations for $u^{\varepsilon}$ and $\psi^{\varepsilon}$ are the same:

$$
\begin{gathered}
-\triangle \psi^{\varepsilon}=\omega^{\varepsilon}, \quad u^{\varepsilon}=\nabla \wedge \psi^{\varepsilon}, \\
\left.\psi^{\varepsilon}\right|_{\partial \Omega}=0 .
\end{gathered}
$$

To solve (8)-(11) further discretization is needed. However we restrict ourselves to consider semi-discrete problems in this paper. Some material on full discretization was presented in [17]. If this scheme is applied to the inviscid step for the NavierStokes equations, then $X_{j_{0}}^{\varepsilon}$ keeps stationary, and the scheme is even simpler.

To prove the consistency, stability, and convergence of the scheme (8)-(11) we need a regular mesh as usual. Let us construct the mesh as follows.

For each point $x \in \partial \Omega$, there is a neighborbood such that $\partial \Omega$ in this neighborhood can be expressed in terms of a smooth function in local coordinates. Let us take a finite set of them, $\left\{\Omega^{(k)}\right\}_{k=1}^{K}$, such that $\bigcup_{k=1}^{K} \Omega^{(k)}$ covers $\partial \Omega$. We thus take a subdomain $\Omega^{(0)} \subset \Omega$ such that $\bigcup_{k=0}^{K} \Omega^{(k)}$ covers $\bar{\Omega}$. For each $\Omega^{(k)}, k=1, \cdots, K$, there is a domain $\tilde{\Omega}$ on the $\xi$-plane $\mathbb{R}^{2}$ such that there is a bijection $F^{(k)}$ defined on $\tilde{\Omega}$ such that $F^{(k)}$ and $\left(F^{(k)}\right)^{-1}$ are smooth and $F^{(k)} \tilde{\Omega}=\Omega^{(k)}$. Moreover we require that the image of $\left\{\xi ; \xi_{2}=0\right\} \cap \tilde{\Omega}$ is $\partial \Omega \cap \Omega^{(k)}$ and $\xi_{2}>0$ corresponds to the interior of $\Omega$, where $\xi=\left(\xi_{1}, \xi_{2}\right)$. We subdivide $\tilde{\Omega}$ into squares with side length $h$ in the following way: Let $j=\left(j_{1}, j_{2}, k\right), j_{1}, j_{2} \in \mathbb{Z}, \tilde{X}_{j}=\left(j_{1} h, j_{2} h\right)$, $\tilde{B}_{j}=\left\{\xi ; j_{1} h-\frac{1}{2} h<\xi_{1}<j_{1} h+\frac{1}{2} h, j_{2} h-\frac{1}{2} h<\xi_{2}<j_{2} h+\frac{1}{2} h\right\}$. Then we define $X_{j}=F^{(k)} \tilde{X}_{j}, B_{j}=F^{(k)} \tilde{B}_{j}$. Thus if $j_{2}=0, X_{j} \in \partial \Omega$, and if $j_{2}>0, X_{j} \in \Omega$. The domain $\Omega^{(0)}$ is subdivided without mapping.

We define functions $\varphi_{k} \in C_{0}^{\infty}\left(\Omega^{(k)}\right)$ such that $\sum_{k=0}^{K} \varphi_{k}(x) \equiv 1$ for $x \in \bar{\Omega}$. Then we define $\omega_{0}^{(k)}=\varphi_{k} \omega_{0}, \alpha_{j}=\omega_{0}^{(k)}\left(X_{j}\right)\left|A\left(\tilde{X}_{j}\right)\right| h^{2}$, where $A\left(\tilde{X}_{j}\right)$ is the Jacobian of $F^{(k)}$ at the point $\tilde{X}_{j}$. Let $J^{(k)}$ be the set of all nodes $X_{j}$ already defined in $\Omega^{(k)}$, and $J^{(0)}$ the set of nodes in $\Omega^{(0)}$. Denote by $J_{1}$ the union $\bigcup_{k=0}^{K} J^{(k)}$. We notice that the meshes overlap each other and some nodes are in the exterior of $\bar{\Omega}$. Let $J=\left\{j \in J_{1} ; X_{j} \in \bar{\Omega}\right\}$. For a given time $t$, if for one index $j$ the distance $\operatorname{dist}\left(X_{j}^{\varepsilon}(t), \partial \Omega\right)<\varepsilon$, then we take the symmetric point of $X_{j}^{\varepsilon}(t)$ at the exterior of $\Omega$ as follows: Let $j=\left(j_{1}, j_{2} . k\right)$; then we define

$$
j^{\prime}=E j=\left(j_{1},-j_{2}, k\right), \quad j_{0}=\left(j_{1}, 0, k\right),
$$

and let $J(t)$ be the set $\left\{j^{\prime}\right\}$. 
For a single mesh the centers of any two neighboring vortex blobs cannot approach each other, but that is not the case now. This is the main difference between this overlapped mesh and a single mesh. Since vortex blobs are applied instead of point vortices, we will see that, however, consistency and stability still hold.

\section{Convergence}

First of all let us introduce some new functions and notations to meet the needs of our convergence proof. Let $u, \omega, \psi$ be the solutions to (1)-(4) and (5)-(7) on the domain $\bar{\Omega} \cap[0, T]$. We extend $\psi$ smoothly so that it is compactly supported in $\left(\bigcup_{k=0}^{K} \Omega^{(k)}\right) \times[0, T]$. From $\psi$ we get extensions of $u$ and $\omega$ by definition. Extending to zero on the whole space, we get an equation for $\omega$ and $u$ :

$$
\frac{\partial \omega}{\partial t}+u \cdot \nabla \omega=F
$$

on $\mathbb{R}^{2} \times[0, T]$. By $(5), F \equiv 0$ in $\bar{\Omega} \times[0, T]$. We define $X_{j}(t)$ and $\alpha_{j}(t)$ by

$$
\begin{aligned}
\frac{d X_{j}}{d t}= & u\left(X_{j}(t), t\right), \quad X_{j}(0)=X_{j}, \quad j \in J_{1}, \\
\frac{d \alpha_{j}}{d t}= & \varphi_{k}\left(X_{j}\right) F\left(X_{j}(t), t\right)\left|A\left(\tilde{X}_{j}\right)\right| h^{2}, \\
& \alpha_{j}(0)=\omega_{0}^{(k)}\left(X_{j}\right)\left|A\left(\tilde{X}_{j}\right)\right| h^{2}, \quad j \in J_{1} .
\end{aligned}
$$

Letting $J_{0}=\left\{j \in J_{1} ; X_{j} \in \partial \Omega\right\}$, we define the $L^{p}$-norm of the error of characteristic curves,

$$
\begin{gathered}
\|e(t)\|_{0, p, h}=\left(\sum_{j \in J} h^{2}\left|X_{j}(t)-X_{j}^{\varepsilon}(t)\right|^{p}\right)^{\frac{1}{p}}+\varepsilon^{\frac{1}{p}}\left(\sum_{j \in J_{0}} h\left|X_{j}(t)-X_{j}^{\varepsilon}(t)\right|^{p}\right)^{\frac{1}{p}}, \\
\|e(t)\|_{0, \infty, h}=\max _{j \in J}\left|X_{j}(t)-X_{j}^{\varepsilon}(t)\right| .
\end{gathered}
$$

Denote $u=G(\omega)$ if $u$ and $\omega$ is related by (6). We decompose the error of velocity into three parts:

$$
u-u^{\varepsilon}=v_{1}+v_{2}+v_{3},
$$

where

$$
\begin{gathered}
v_{1}=u-G\left(\omega(\cdot, t) * \zeta_{\varepsilon}\right), \\
v_{2}=G\left(\omega(\cdot, t) * \zeta_{\varepsilon}-\sum_{j \in J_{1}} \alpha_{j}(t) \zeta_{\varepsilon}\left(\cdot-X_{j}(t)\right)\right), \\
v_{3}=G\left(\sum_{j \in J_{1}} \alpha_{j}(t) \zeta_{\varepsilon}\left(\cdot-X_{j}(t)\right)-\sum_{j \in J \cup J(t)} \alpha_{j}^{\varepsilon} \zeta_{\varepsilon}\left(\cdot-X_{j}^{\varepsilon}(t)\right)\right) .
\end{gathered}
$$

Let us estimate the semi-norm $|\cdot|_{l, p}$ of $v_{i}, i=1,2,3$. We will always denote by $C$ a generic positive constant which is independent of the mesh parameter $h$ and $\varepsilon$, and by $C_{1}, C_{2}, \cdots$ some particular positive constants. 
Lemma 1. If $\zeta \in L^{\infty}\left(\mathbb{R}^{2}\right)$ and

$$
\int_{\mathbb{R}^{2}} x^{\alpha} \zeta(x) d x=0, \quad|\alpha|=1,
$$

then for integers $l \geq 0$

$$
\left|v_{1}(\cdot, t)\right|_{l, p, \Omega} \leq C \varepsilon^{2}, \quad 1 \leq p \leq+\infty .
$$

Proof. See [18] for details.

Lemma 2. Let $p>1, l \geq 0$ and $\zeta \in W^{m+l-1, \infty}\left(\mathbb{R}^{2}\right)$ for $m \geq 1$. Then for any constant $r \in[1,2)$ and an arbitrary integer $N \geq 3$, we have

$$
\left|v_{2}(\cdot, t)\right|_{l, p, \Omega} \leq C\left(\left(1+\frac{h}{\varepsilon}\right)^{\frac{2}{r}} \frac{h^{m}}{\varepsilon^{m+l-1}}+\frac{h^{N}}{\varepsilon^{N+l-1}}\right) .
$$

Proof. Since $v_{2}$ is a linear combination with respect to $k$ 's, it would suffice to consider one $k$ only. We define characteristic curves for all initial points, $\xi(\tau ; x, t)$ by

$$
\begin{gathered}
\frac{d \xi}{d t}=u(\xi(\tau ; x, t), \tau), \\
\xi(t ; x, t)=x .
\end{gathered}
$$

Then we consider

$$
v_{2}^{(k)}=G\left(\left(\varphi_{k}(\xi(0 ; \cdot, t)) \omega(\cdot, t)\right) * \zeta_{\varepsilon}-\sum_{j \in J^{(k)}} \alpha_{j}(t) \zeta_{\varepsilon}\left(\cdot-X_{j}(t)\right)\right) .
$$

Since the mapping $x \rightarrow \xi$ defined by (20) and (21) is measure preserving, we have

$$
\begin{aligned}
\left(\left(\varphi_{k}(\xi(0 ; \cdot, t)) \omega(\cdot, t)\right) * \zeta_{\varepsilon}\right)(x) \\
\quad=\int \zeta_{\varepsilon}(x-y) \varphi_{k}(\xi(0 ; y, t)) \omega(y, t) d y \\
\quad=\int \zeta_{\varepsilon}(x-\xi(t ; \eta, 0)) \varphi_{k}(\eta) \omega(\xi(t ; \eta, 0), t) d \eta \\
\quad=\int \zeta_{\varepsilon}\left(x-\xi\left(t ; F^{(k)} z, 0\right)\right) \varphi_{k}\left(F^{(k)} z\right) \omega\left(\xi\left(t ; F^{(k)} z, 0\right), t\right)|A(z)| d z .
\end{aligned}
$$

We define

$$
g(x, z, t)=\partial_{x}^{\gamma} \zeta_{\varepsilon}\left(x-\xi\left(t ; F^{(k)} z, 0\right)\right) \varphi_{k}\left(F^{(k)} z\right) \omega\left(\xi\left(t ; F^{(k)} z, 0\right), t\right)|A(z)| ;
$$

then by the equations (12)-(14) we have

$$
\alpha_{j}(t)=\varphi_{k}\left(X_{j}\right) \omega\left(\xi\left(t ; X_{j}, 0\right), t\right)\left|A\left(\tilde{X}_{j}\right)\right| h^{2},
$$

but $X_{j}=F^{(k)} \tilde{X}_{j}$, so

$$
g\left(x, \tilde{X}_{j}, t\right)=\alpha_{j}(t) \partial^{\gamma} \zeta_{\varepsilon}\left(x-X_{j}(t)\right) / h^{2} .
$$

Consequently

$$
\begin{aligned}
& \partial^{\gamma}\left(\left(\left(\varphi_{k}(\xi(0 ; \cdot, t)) \omega(\cdot, t)\right) * \zeta_{\varepsilon}\right)(x)-\sum_{j \in J^{(k)}} \alpha_{j}(t) \zeta_{\varepsilon}\left(x-X_{j}(t)\right)\right) \\
& \quad=\int g(x, z, t) d z-\sum_{j \in J^{(k)}} g\left(x, \tilde{X}_{j}, t\right) h^{2} .
\end{aligned}
$$


Let $|\gamma|=l$; then, following the same lines as the proof in [18], we get the estimation (19) for $v_{2}^{(k)}$, which completes the proof.

Lemma 3. If there are constants $C_{1}, C_{2}$ such that $\left|\nabla u^{\varepsilon}\right| \leq C_{1}, h \leq C_{2} \varepsilon$, and if $\zeta \in W^{l, \infty}\left(\mathbb{R}^{2}\right)$ for $l \geq 0$, then for sufficiently small $\varepsilon$ we have

$$
\left|v_{3}(\cdot, t)\right|_{l, p, \Omega} \leq \frac{C}{\varepsilon^{l}}\left(1+\frac{\|e(t)\|_{0, \infty, h}}{\varepsilon}\right)^{\frac{2}{q}}\left(\|e(t)\|_{0, p, h}+\varepsilon^{2+\frac{1}{p}}\right),
$$

where $p>1, \frac{1}{p}+\frac{1}{q}=1$.

Proof. Since $|\nabla u| \leq C$ and $\left|\nabla u^{\varepsilon}\right| \leq C_{1}$, there is a constant $C_{0}>1$ such that if $\operatorname{dist}\left(X_{j}, \bar{\Omega}\right)>C_{0} \varepsilon$, then supp $\zeta_{\varepsilon}\left(\cdot-X_{j}(t)\right)$ and $\operatorname{supp} \zeta_{\varepsilon}\left(\cdot-X_{j}^{\varepsilon}(t)\right)$ have no intersection with $\bar{\Omega}$. We define $J_{2}=\left\{j ; 0<\operatorname{dist}\left(X_{j}, \bar{\Omega}\right) \leq C_{0} \varepsilon\right\}$; then

$$
v_{3}=G\left(\sum_{j \in J \cup J_{2}}\left(\alpha_{j}(t) \zeta_{\varepsilon}\left(\cdot-X_{j}(t)\right)-\alpha_{j}^{\varepsilon} \zeta_{\varepsilon}\left(\cdot-X_{j}^{\varepsilon}(t)\right)\right)\right) \text {. }
$$

Let

$$
v_{3}=v_{3,1}+v_{3,2}+v_{3,3},
$$

where

$$
\begin{gathered}
v_{3,1}=G\left(\sum_{j \in J}\left(\alpha_{j}(t) \zeta_{\varepsilon}\left(\cdot-X_{j}(t)\right)-\alpha_{j}^{\varepsilon} \zeta_{\varepsilon}\left(\cdot-X_{j}^{\varepsilon}(t)\right)\right),\right. \\
v_{3,2}=G\left(\sum_{j \in J_{2}}\left(\alpha_{j}(t)-\alpha_{j}^{\varepsilon}\right) \zeta_{\varepsilon}\left(\cdot-X_{j}(t)\right)\right), \\
v_{3,3}=G\left(\sum_{j \in J_{2}} \alpha_{j}^{\varepsilon}\left(\zeta_{\varepsilon}\left(\cdot-X_{j}(t)\right)-\zeta_{\varepsilon}\left(\cdot-X_{j}^{\varepsilon}(t)\right)\right)\right) .
\end{gathered}
$$

We notice that $\alpha_{j}(t)=\alpha_{j}^{\varepsilon}=\alpha_{j}$ for $j \in J$; hence

$$
\left|v_{3,1}\right|_{l, p, \Omega} \leq \frac{C}{\varepsilon^{l}}\left(1+\frac{\|e(t)\|_{0, \infty, h}}{\varepsilon}\right)^{\frac{2}{q}}\|e(t)\|_{0, p, h} .
$$

The proof of (22) is essentially the same as that in [18], except we notice that the estimate for the cardinal number, $\operatorname{card} J_{x}$, is multiplied by a constant $K$.

We turn now to estimating $v_{3,2}$. Since $F \equiv 0$ in $\bar{\Omega} \times[0, T]$ and $F$ is smooth, we have from (14) that

$$
\alpha_{j}(t)=\omega_{0}^{(k)}\left(X_{j}\right)\left|A\left(\tilde{X}_{j}\right)\right| h^{2}+O(\varepsilon) h^{2} .
$$

Also,

$$
\alpha_{j}^{\varepsilon}=\alpha_{E j}^{\varepsilon}=\omega_{0}^{(k)}\left(X_{E j}\right)\left|A\left(\tilde{X}_{E j}\right)\right| h^{2} .
$$

Because $j \in J_{2}$, dist $\left(\tilde{X}_{j}, \tilde{X}_{E j}\right) \leq C \varepsilon$; hence

$$
\left|\alpha_{j}(t)-\alpha_{j}^{\varepsilon}\right| \leq C \varepsilon h^{2} .
$$

For a fixed point $x$ we define $J_{x}=\left\{j \in J_{2} ; x \in \operatorname{supp} \zeta_{\varepsilon}\left(\cdot-X_{j}(t)\right)\right\}$. Let $B_{j}(t)$ be the image of $B_{j}$ under the mapping $(20),(21)$; then the diameter of $B_{j}(t)$ is bounded 
by $C_{3} h$. We construct a disk with center $x$ and radius $\varepsilon+C_{3} h$; then, if $j \in J_{x}$, $B_{j}(t)$ is contained in the disk. Since the mapping (20), (21) is measure preserving, we have

$$
\operatorname{card} J_{x} \leq \frac{\pi(\varepsilon+C h)^{2} K}{h^{2}} \leq C\left(1+\frac{\varepsilon}{h}\right)^{2}
$$

Let

$$
g=\sum_{j \in J_{2}}\left(\alpha_{j}(t)-\alpha_{j}^{\varepsilon}\right) \zeta_{\varepsilon}\left(x-X_{j}(t)\right)
$$

then by [18], Lemma 5.1,

$$
|g|_{l-1, p} \leq C\left(1+\frac{\varepsilon}{h}\right)^{\frac{2}{q}}\left(\sum_{j \in J_{2}}\left\|\left(\alpha_{j}(t)-\alpha_{j}^{\varepsilon}(t)\right) \partial^{\gamma} \zeta_{\varepsilon}\left(\cdot-X_{j}(t)\right)\right\|_{0, p}^{p}\right)^{\frac{1}{p}},
$$

where $l \geq 1,|\gamma|=l-1$. We consider the set

$$
D=\left\{x ; 0<\operatorname{dist}(x, \bar{\Omega}) \leq C_{0} \varepsilon+C_{3} h\right\} ;
$$

then area $D \leq C\left(C_{0} \varepsilon+C_{3} h\right)$, and it is easy to see that

$$
\text { card } J_{2} \leq \frac{C\left(C_{0} \varepsilon+C_{3} h\right)}{h^{2}} \leq C\left(\frac{1}{h}+\frac{\varepsilon}{h^{2}}\right) .
$$

Besides, we have

$$
\left\|\partial^{\gamma} \zeta_{\varepsilon}\right\|_{0, p} \leq \frac{C}{\varepsilon^{l-1+\frac{2}{q}}}
$$

therefore

$$
\begin{aligned}
|g|_{l-1, p} & \leq C\left(1+\frac{\varepsilon}{h}\right)^{\frac{2}{q}}\left(\frac{1}{h}+\frac{\varepsilon}{h^{2}}\right)^{\frac{1}{p}} \varepsilon h^{2} \frac{1}{\varepsilon^{l-1+\frac{2}{q}}} \\
& =\frac{C}{\varepsilon^{l}} \varepsilon^{2+\frac{1}{p}}\left(1+\frac{h}{\varepsilon}\right)^{1+\frac{1}{q}} .
\end{aligned}
$$

It remains to consider the case of $l=0$. Let $g^{(k)}=\varphi_{k} g$. We construct local coordinates $(n, \tau)$ on $\Omega^{(k)}$ and let $n=0$ correspond to $\partial \Omega$. Let $\Omega_{d}=\{(n, \tau) ; 0<$ $n<d,|\tau|<C\}$. We estimate $\left|g^{(k)}\right|_{-1, p, \Omega_{d}}$. Let $\tilde{g}=\int_{d}^{n} g d n$. For $\varphi \in C_{0}^{\infty}\left(\Omega_{d}\right)$ we have

$$
\begin{aligned}
\int_{\Omega_{d}} \varphi g d n d \tau & =\int d \tau \int \varphi \frac{\partial \tilde{g}}{\partial n} d n=-\int d \tau \int \tilde{g} \frac{\partial \varphi}{\partial n} d n \\
& \leq\|\tilde{g}\|_{0, p}\left\|\frac{\partial \varphi}{\partial n}\right\|_{0, q} .
\end{aligned}
$$

We have

$$
\|g\|_{0, \infty} \leq \sup \left|\left(\alpha_{j}(t)-\alpha_{j}^{\varepsilon}\right) \zeta_{\varepsilon}\left(x-X_{j}(t)\right)\right| \operatorname{card} J_{x} \leq C \varepsilon\left(1+\frac{h}{\varepsilon}\right)^{2} .
$$

The support of $g$ is contained in $\{(n, \tau) ; 0 \leq n \leq C \varepsilon\}$; hence

$$
\|\tilde{g}\|_{0, p} \leq \varepsilon^{2+\frac{1}{p}}\left(1+\frac{h}{\varepsilon}\right)^{2},
$$


and consequently

$$
\|g\|_{-1, p} \leq C \varepsilon^{2+\frac{1}{p}}\left(1+\frac{h}{\varepsilon}\right)^{2} .
$$

By the $L^{p}$-norm estimate [2], [3] we get

$$
\left|v_{3,2}\right|_{l, p, \Omega} \leq \frac{C}{\varepsilon^{l}} \varepsilon^{2+\frac{1}{p}}, \quad l \geq 0 .
$$

Finally let us estimate $v_{3,3}$. At the time $t=0$ we have

$$
X_{j}(0)=X_{j}=F^{(k)} \tilde{X}_{j}
$$

and

$$
\begin{aligned}
X_{j}^{\varepsilon}(0) & =2 X_{j_{0}}^{\varepsilon}(0)-X_{E j}^{\varepsilon}(0) \\
& =2 X_{j_{0}}-X_{E j}=2 F^{(k)} \tilde{X}_{j_{0}}-F^{(k)} \tilde{X}_{E j} .
\end{aligned}
$$

Applying Taylor's expansion about the point $\tilde{X}_{E j}$ we get

$$
\begin{gathered}
F^{(k)} \tilde{X}_{j}=F^{(k)} \tilde{X}_{E j}+D F^{(k)}\left(\tilde{X}_{j}-\tilde{X}_{E j}\right)+O\left(\varepsilon^{2}\right), \\
F^{(k)} \tilde{X}_{j_{0}}=F^{(k)} \tilde{X}_{E j}+D F^{(k)}\left(\tilde{X}_{j_{0}}-\tilde{X}_{E j}\right)+O\left(\varepsilon^{2}\right) .
\end{gathered}
$$

But we have

$$
\tilde{X}_{j}-\tilde{X}_{E j}=2\left(\tilde{X}_{j_{0}}-\tilde{X}_{E j}\right)
$$

and hence

$$
\begin{aligned}
X_{j}(0)-X_{j}^{\varepsilon}(0) & =\left(X_{j}-X_{E j}\right)-2\left(X_{j_{0}}-X_{E j}\right) \\
& =\left(F^{(k)} \tilde{X}_{j}-F^{(k)} \tilde{X}_{E j}\right)-2\left(F^{(k)} \tilde{X}_{j_{0}}-F^{(k)} \tilde{X}_{E j}\right) \\
& =O\left(\varepsilon^{2}\right) .
\end{aligned}
$$

Applying Taylor's expansion to the function $u$, we get

$$
\begin{gathered}
u\left(X_{j_{0}}(t), t\right)=u\left(X_{E j}(t), t\right)+D u\left(X_{j_{0}}(t)-X_{E j}(t)\right)+O\left(\varepsilon^{2}\right), \\
u\left(X_{j}(t), t\right)=u\left(X_{E j}(t), t\right)+D u\left(X_{j}(t)-X_{E j}(t)\right)+O\left(\varepsilon^{2}\right) .
\end{gathered}
$$

Then by the equation (13) we get

$$
\begin{gathered}
\frac{d\left(X_{j_{0}}(t)-X_{E j}(t)\right)}{d t}=D u\left(X_{j_{0}}(t)-X_{E j}(t)\right)+O\left(\varepsilon^{2}\right), \\
\frac{d\left(X_{j}(t)-X_{E j}(t)\right)}{d t}=D u\left(X_{j}(t)-X_{E j}(t)\right)+O\left(\varepsilon^{2}\right) .
\end{gathered}
$$

Let $f(t)=\left(X_{j}(t)-X_{E j}(t)\right)-2\left(X_{j_{0}}(t)-X_{E j}(t)\right)$; then

$$
\frac{d f}{d t}=D u \cdot f+O\left(\varepsilon^{2}\right)
$$

By (24) $f(0)=O\left(\varepsilon^{2}\right)$; hence $f(t)=O\left(\varepsilon^{2}\right)$. Also, we have

$$
X_{j}^{\varepsilon}(t)=2 X_{j_{0}}^{\varepsilon}(t)-X_{E j}^{\varepsilon}(t)
$$

therefore

$$
X_{j}(t)-X_{j}^{\varepsilon}(t)=2\left(X_{j_{0}}(t)-X_{j_{0}}^{\varepsilon}(t)\right)-\left(X_{E j}(t)-X_{E j}^{\varepsilon}(t)\right)+O\left(\varepsilon^{2}\right) .
$$


Consequently

$$
\begin{aligned}
-v_{3,3} & =G\left(\sum_{j \in J_{2}} \alpha_{j}^{\varepsilon} \int_{0}^{1} D \zeta_{\varepsilon}\left(x-X_{j}(t)+\theta\left(X_{j}(t)-X_{j}^{\varepsilon}(t)\right)\right) d \theta\left(X_{j}(t)-X_{j}^{\varepsilon}(t)\right)\right) \\
& =w_{1}+w_{2}+w_{3},
\end{aligned}
$$

where

$$
\begin{gathered}
w_{1}=2 G\left(\sum _ { j \in J _ { 2 } } \alpha _ { j } ^ { \varepsilon } \int _ { 0 } ^ { 1 } D \zeta _ { \varepsilon } \left(x-X_{j}(t)\right.\right. \\
\left.\left.+\theta\left(X_{j}(t)-X_{j}^{\varepsilon}(t)\right)\right) d \theta\left(X_{j_{0}}(t)-X_{j_{0}}^{\varepsilon}(t)\right)\right), \\
-w_{2}=G\left(\sum _ { j \in J _ { 2 } } \alpha _ { j } ^ { \varepsilon } \int _ { 0 } ^ { 1 } D \zeta _ { \varepsilon } \left(x-X_{j}(t)\right.\right. \\
\left.\left.+\theta\left(X_{j}(t)-X_{j}^{\varepsilon}(t)\right)\right) d \theta\left(X_{E j}(t)-X_{E j}^{\varepsilon}(t)\right)\right), \\
w_{3}=G\left(O\left(\varepsilon^{2}\right) \sum_{j \in J_{2}} \alpha_{j}^{\varepsilon} \int_{0}^{1} D \zeta_{\varepsilon}\left(x-X_{j}(t)+\theta\left(X_{j}(t)-X_{j}^{\varepsilon}(t)\right)\right) d \theta\right) .
\end{gathered}
$$

The estimate for $w_{2}$ is the same as $v_{3,1}$. Thus we have

$$
\left|w_{2}\right|_{l, p, \Omega} \leq \frac{C}{\varepsilon^{l}}\left(1+\frac{\|e(t)\|_{0, \infty, h}}{\varepsilon}\right)^{\frac{2}{q}}\|e(t)\|_{0, p, h} .
$$

The estimate for $w_{3}$ is the same as $v_{3,2}$, except that

$$
\operatorname{card} J_{x} \leq C\left(1+\frac{\varepsilon}{h}+\frac{\|e(t)\|_{0, \infty, h}}{h}\right)^{2} ;
$$

therefore

$$
\left|w_{3}\right|_{l, p, \Omega} \leq \frac{C}{\varepsilon^{l}} \varepsilon^{2+\frac{1}{p}}\left(1+\frac{\|e(t)\|_{0, \infty, h}}{\varepsilon}\right)^{2} .
$$

It remains to estimate $w_{1}$. By analogy to (25) we get

$$
\left|w_{1}\right|_{l, p, \Omega} \leq \frac{C}{\varepsilon^{l}}\left(1+\frac{\|e(t)\|_{0, \infty, h}}{\varepsilon}\right)^{\frac{2}{q}}\left(\sum_{j \in J_{2}} h^{2}\left|X_{j_{0}}(t)-X_{j_{0}}^{\varepsilon}(t)\right|^{p}\right)^{\frac{1}{p}} .
$$

We notice that $j_{0}=\left(j_{1}, 0, k\right)$ and $j=\left(j_{1}, j_{2}, k\right)$; hence, for fixed $j_{0}$, card $\{j\}=$ card $\left\{j_{2}\right\} \leq C\left(1+\frac{\varepsilon}{h}\right)$. Then

$$
\sum_{j \in J_{2}} h^{2}\left|X_{j_{0}}(t)-X_{j_{0}}^{\varepsilon}(t)\right|^{p} \leq C\left(1+\frac{\varepsilon}{h}\right) h \sum_{j_{0} \in J_{0}} h\left|X_{j_{0}}(t)-X_{j_{0}}^{\varepsilon}(t)\right|^{p} .
$$

By the definition (15) we have

$$
\left|w_{1}\right|_{l, p, \Omega} \leq \frac{C}{\varepsilon^{l}}\left(1+\frac{\|e(t)\|_{0, \infty, h}}{\varepsilon}\right)^{\frac{2}{q}}\|e(t)\|_{0, p, h} .
$$

The combination of (22), (23), (25)-(27) gives the desired estimate. 
We now estimate $\|e(t)\|_{0, p, h}$. By (8) and (13) we have

$$
X_{j}(t)-X_{j}^{\varepsilon}(t)=\int_{0}^{t}\left(u\left(X_{j}(s), s\right)-u^{\varepsilon}\left(X_{j}^{\varepsilon}(s), s\right)\right) d s=I_{j}^{(1)}+I_{j}^{(2)},
$$

where

$$
\begin{gathered}
I_{j}^{(1)}=\int_{0}^{t}\left(u-u^{\varepsilon}\right)\left(X_{j}^{\varepsilon}(s), s\right) d s \\
I_{j}^{(2)}=\int_{0}^{t}\left(u^{\varepsilon}\left(X_{j}(s), s\right)-u^{\varepsilon}\left(X_{j}^{\varepsilon}(s), s\right)\right) d s .
\end{gathered}
$$

Lemma 4. We assume that the moment condition (17) holds. If $\left|\nabla u^{\varepsilon}\right| \leq C_{1}$, $\zeta \in W^{m+1, \infty}\left(\mathbb{R}^{2}\right), m \geq 2, p>2$, and

$$
C_{4}^{-1} \varepsilon^{a} \leq h \leq C_{4} \varepsilon^{\frac{m+1}{m}},
$$

where $\frac{p}{2}>a \geq \frac{m+1}{m}$, then we have

$$
\|e(t)\|_{0, p, h} \leq C \varepsilon^{2}
$$

for $t \in[0, T]$, provided $\varepsilon$ is sufficiently small.

Proof. We start by estimating the first term of (15). By Lemmas 1, 2, 3 we have

$$
\begin{aligned}
\left|u-u^{\varepsilon}\right|_{l, p, \Omega} & \leq C\left\{\varepsilon^{2}+\left(1+\frac{h}{\varepsilon}\right)^{\frac{2}{r}} \frac{h^{m}}{\varepsilon^{m+l-1}}+\frac{h^{N}}{\varepsilon^{N+l-1}}\right. \\
& \left.+\frac{1}{\varepsilon^{l}}\left(1+\frac{\|e(t)\|_{0, \infty, h}}{\varepsilon}\right)^{\frac{2}{q}}\left(\|e(t)\|_{0, p, h}+\varepsilon^{2+\frac{1}{p}}\right)\right\},
\end{aligned}
$$

where $\frac{1}{p}+\frac{1}{q}=1, N$ is large enough, and $l=0,1,2$. By [13]

$$
\left(\sum_{j \in J} h^{2}\left|\left(u-u^{\varepsilon}\right)\left(X_{j}(t), t\right)\right|^{p}\right)^{\frac{1}{p}} \leq C K^{\frac{1}{p}}\left(\left\|u-u^{\varepsilon}\right\|_{0, p, \Omega}+h\left|u-u^{\varepsilon}\right|_{1, p, \Omega}\right) .
$$

Due to (30) and the assumption we obtain

$$
\begin{aligned}
& \left(\sum_{j \in J} h^{2}\left|I_{j}^{(1)}\right|^{p}\right)^{\frac{1}{p}} \\
& \leq C \int_{0}^{t}\left\{\varepsilon^{2}+\left(1+\frac{\|e(s)\|_{0, \infty, h}}{\varepsilon}\right)^{\frac{2}{q}}\left(\|e(s)\|_{0, p, h}+\varepsilon^{2+\frac{1}{p}}\right)\right\} d s .
\end{aligned}
$$

By the assumption we have

$$
\begin{aligned}
\left(\sum_{j \in J} h^{2}\left|I_{j}^{(2)}\right|^{p}\right)^{\frac{1}{p}} & \leq C \int_{0}^{t}\left(\sum_{j \in J} h^{2}\left|X_{j}(s)-X_{j}^{\varepsilon}(s)\right|^{p}\right)^{\frac{1}{p}} d s \\
& \leq C \int_{0}^{t}\|e(s)\|_{0, p, h} d s .
\end{aligned}
$$


We now estimate the second term of (15). Analogously to (32),

$$
\varepsilon^{\frac{1}{p}}\left(\sum_{j \in J_{0}} h\left|I_{j}^{(2)}\right|^{p}\right)^{\frac{1}{p}} \leq C \int_{0}^{t}\|e(s)\|_{0, p, h} d s .
$$

Let

$$
f(\eta)=\left(u-u^{\varepsilon}\right)\left(\xi\left(t ; F^{(k)} \eta, 0\right), t\right) ;
$$

then by [13] we have analogously

$$
\left(\sum_{j \in J_{0}} h\left|f\left(\tilde{X}_{j}\right)\right|^{p}\right)^{\frac{1}{p}} \leq C K^{\frac{1}{p}}\left(\|f\|_{0, p, \mathbb{R}}+h|f|_{1, p, \mathbb{R}}\right) .
$$

We set $\eta=\varepsilon \eta^{\prime}$ and apply the trace theorem [1] to get

$$
\begin{gathered}
\|f(\varepsilon \cdot)\|_{0, p, \mathbb{R}} \leq C\|f(\varepsilon \cdot)\|_{1, p, \mathbb{R}^{2}}, \\
|f(\varepsilon \cdot)|_{1, p, \mathbb{R}} \leq C\left(|f(\varepsilon \cdot)|_{1, p, \mathbb{R}^{2}}+|f(\varepsilon \cdot)|_{2, p, \mathbb{R}^{2}}\right) .
\end{gathered}
$$

Therefore

$$
\begin{gathered}
\|f\|_{0, p, \mathbb{R}} \leq \varepsilon^{-\frac{1}{p}} C\left(\|f\|_{0, p, \mathbb{R}^{2}}+\varepsilon|f|_{1, p, \mathbb{R}^{2}}\right) \\
|f|_{1, p, \mathbb{R}} \leq \varepsilon^{-\frac{1}{p}} C\left(|f|_{1, p, \mathbb{R}^{2}}+\varepsilon|f|_{2, p, \mathbb{R}^{2}}\right) .
\end{gathered}
$$

Consequently

$$
\left(\sum_{j \in J_{0}} h\left|f\left(\tilde{X}_{j}\right)\right|^{p}\right)^{\frac{1}{p}} \leq \varepsilon^{-\frac{1}{p}} C\left(\|f\|_{0, p, \mathbb{R}^{2}}+\varepsilon|f|_{1, p, \mathbb{R}^{2}}+\varepsilon h|f|_{2, p, \mathbb{R}^{2}}\right) .
$$

Therefore

$$
\begin{aligned}
& \varepsilon^{\frac{1}{p}}\left(\sum_{j \in J_{0}} h\left|I_{j}^{(1)}\right|^{p}\right)^{\frac{1}{p}} \leq C \int_{0}^{t} \varepsilon^{\frac{1}{p}}\left(\sum_{j \in J_{0}} h\left|f\left(\tilde{X}_{j}\right)\right|^{p}\right)^{\frac{1}{p}} d s \\
& \leq C \int_{0}^{t}\left(\|f\|_{0, p, \mathbb{R}^{2}}+\varepsilon|f|_{1, p, \mathbb{R}^{2}}+\varepsilon h|f|_{2, p, \mathbb{R}^{2}}\right) d s .
\end{aligned}
$$

Since $\left(F^{(k)}\right)^{-1}$ is smooth, we get

$$
\begin{aligned}
& \varepsilon^{\frac{1}{p}}\left(\sum_{j \in J_{0}} h\left|I_{j}^{(1)}\right|^{p}\right)^{\frac{1}{p}} \\
& \quad \leq C \int_{0}^{t}\left(\left\|u-u^{\varepsilon}\right\|_{0, p, \mathbb{R}^{2}}+\varepsilon\left|u-u^{\varepsilon}\right|_{1, p, \mathbb{R}^{2}}+\varepsilon h\left|u-u^{\varepsilon}\right|_{2, p, \mathbb{R}^{2}}\right) d s
\end{aligned}
$$


It is analogous to (31) that

$$
\begin{aligned}
& \varepsilon^{\frac{1}{p}}\left(\sum_{j \in J_{0}} h\left|I_{j}^{(1)}\right|^{p}\right)^{\frac{1}{p}} \\
& \quad \leq C \int_{0}^{t}\left\{\varepsilon^{2}+\left(1+\frac{\|e(s)\|_{0, \infty, h}}{\varepsilon}\right)^{\frac{2}{q}}\left(\|e(s)\|_{0, p, h}+\varepsilon^{2+\frac{1}{p}}\right)\right\} d s .
\end{aligned}
$$

The combination of (31)-(34) gives

$$
\begin{aligned}
& \|e(t)\|_{0, p, h} \\
& \leq C \int_{0}^{t}\left\{\varepsilon^{2}+\left(1+\frac{\|e(s)\|_{0, \infty, h}}{\varepsilon}\right)^{\frac{2}{q}}\left(\|e(s)\|_{0, p, h}+\varepsilon^{2+\frac{1}{p}}\right)\right\} d s .
\end{aligned}
$$

We claim that

$$
\|e(t)\|_{0, p . h} \leq C_{5} \varepsilon^{2}
$$

where $C_{5}$ is a constant to be determined. Since $\|e(0)\|_{0, p, h}=0$ and $\|e(t)\|_{0, p, h}$ is continuous, (36) holds on an interval $\left[0, T_{*}\right]$. By $(28)$

$$
\begin{aligned}
\|e(t)\|_{0, \infty, h} & \leq h^{-\frac{2}{p}}\|e(t)\|_{0, p, h} \\
& \leq C_{4}^{\frac{2}{p}} \varepsilon^{-\frac{2 a}{p}}\|e(t)\|_{0, p, h} \leq C_{4}^{\frac{2}{p}} C_{5} \varepsilon^{2-\frac{2 a}{p}} .
\end{aligned}
$$

By the assumption $p>2 a$ we have on $\left[0, T_{*}\right]$ that

$$
\left(1+\frac{1}{\varepsilon}\|e(t)\|_{0, \infty, h}\right)^{\frac{2}{q}} \leq C_{6}
$$

where

$$
C_{6} \geq\left(1+C_{4}^{\frac{2}{p}} C_{5} \varepsilon^{2-\frac{2 a}{p}}\right)^{\frac{2}{q}}
$$

(35) is reduced to

$$
\|e(t)\|_{0, p, h} \leq C_{7} \int_{0}^{t}\left\{\varepsilon^{2}+\|e(s)\|_{0, p, h}\right\} d s .
$$

Applying Gronwall's inequality, we get

$$
\|e(t)\|_{0, p, h} \leq C_{7} t e^{C_{7} t} \varepsilon^{2} .
$$

First we take a constant $C_{6}>1$, then we set $C_{5}=C_{7} T e^{C_{7} T}$, then we take $\varepsilon_{0}$ sufficiently small that (37) holds for $\varepsilon \leq \varepsilon_{0}$. If $T_{*}<T$, then by (38) we have

$$
\|e(t)\|_{0, p, h}<C_{5} \varepsilon^{2}
$$

which means the interval $\left[0, T_{*}\right]$ can be extended. Thus $T=T_{*}$.

Corollary 1. Under the conditions of Lemma 4, (29) holds for all $\varepsilon>0$, and the condition $a<\frac{p}{2}$ is not necessary.

In fact we can take $p$ large enough so that $p>2 a$, and then by Lemma 4 we have (29). For $r \in[1, p)$, we can apply the Hölder inequality to get

$$
\|e(t)\|_{0, r, h} \leq C\|e(t)\|_{0, p, h},
$$


because $\Omega$ is bounded. Besides, if $\varepsilon>\varepsilon_{0}$, we have

$$
\|e(t)\|_{0, p, h} \leq C \varepsilon_{0}^{-2} \varepsilon^{2},
$$

since $\|e(t)\|_{0, p, h}$ is bounded.

Finally, let us prove the convergence theorem.

Theorem 1. If the moment condition (17) holds, $\zeta \in W^{m+1, \infty}\left(\mathbb{R}^{2}\right)$ for $m \geq 2$, $p \in[1, \infty)$, and if the condition (28) holds for $a \geq \frac{m+1}{m}$, then

$$
\begin{gathered}
\left|\nabla u^{\varepsilon}\right| \leq C, \quad x \in \bar{\Omega}, \\
\left\|u-u^{\varepsilon}\right\|_{0, p}+\|e(t)\|_{0, p, h} \leq C \varepsilon^{2},
\end{gathered}
$$

where $t \in[0, T]$.

Proof. We take $p>2$ and notice that

$$
\left\|\left(u-u^{\varepsilon}\right)(\cdot, 0)\right\|_{2, p, \Omega}=\left\|\left(v_{1}+v_{2}+v_{3,2}+w_{3}\right)(\cdot, 0)\right\|_{2, p, \Omega} \leq C,
$$

which gives

$$
\left\|\left(u-u^{\varepsilon}\right)(\cdot, 0)\right\|_{1, \infty, \Omega} \leq C_{8} .
$$

Enlarge $C_{8}$ if necessary so that

$$
\left\|\left(v_{1}+v_{2}\right)(\cdot, t)\right\|_{1, \infty, \Omega} \leq C_{8} .
$$

Let

$$
C_{1}=\max _{(x, t) \in \bar{\Omega} \times[0, T]}|\nabla u|+C_{8}+1 ;
$$

then $\left|\nabla u^{\varepsilon}\right| \leq C_{1}$ on a certain interval $\left[0, T_{*}\right]$. By Lemma 4 and Corollary 1

$$
\|e(t)\|_{0, p, h} \leq C \varepsilon^{2} .
$$

By Lemma 3

$$
\left|v_{3}\right|_{l, p, \Omega} \leq C \varepsilon^{2-l} .
$$

We take a constant $s$ such that $2 / p<s<1$; then, by the interpolation inequality [1],

$$
\left\|v_{3}\right\|_{1, \infty, \Omega} \leq C\left\|v_{3}\right\|_{1, p, \Omega}^{1-s}\left\|v_{3}\right\|_{2, p, \Omega}^{s} \leq C \varepsilon^{1-s} .
$$

Taking $\varepsilon$ small enough, we have

$$
\left\|v_{3}\right\|_{1, \infty, \Omega}<1 .
$$

By (41), $\left|\nabla u^{\varepsilon}\right|<C_{1}$. Using continuous extension we get (39) for $t \in[0, T]$ and small $\varepsilon$. Then $(40)$ with large $p$, small $\varepsilon$ follows from Lemmas 1, 2, 3, 4. The restriction for $p, \varepsilon$ can be loosened via the argument of Corollary 1. 


\section{NumericAl EXAMPLE}

Let $\Omega$ be a unit disk. We consider the initial-boundary value problem,

$$
\begin{gathered}
\frac{\partial u}{\partial t}+(u \cdot \nabla) u+\frac{1}{\rho} \nabla p=f, \\
\nabla \cdot u=0, \\
\left.u \cdot n\right|_{\partial \Omega}=0, \\
\left.u\right|_{t=0}=u_{0} .
\end{gathered}
$$

We take the stream function,

$$
\psi=\left(1-|x|^{2}\right)\left(x_{1}+x_{2}\right) e^{-t}
$$

then we get the velocity $u$ and vorticity $\omega$ by direct calculation, and then we get the initial condition for the vorticity,

$$
\left.\omega\right|_{t=0}=8\left(x_{1}+x_{2}\right) .
$$

Upon substituting $u$ and $\omega$ into the equation

$$
\frac{\partial \omega}{\partial t}+u \cdot \nabla \omega=F
$$

we get

$$
F=-8\left(x_{1}+x_{2}\right) e^{-t}+16\left(x_{1}^{2}-x_{2}^{2}\right) e^{-2 t} .
$$

We will compare the approximate solutions to this exact solution.

The first scheme (A) is a simple one without boundary treatment. We subdivide $\mathbb{R}^{2}$ into squares with side length $h$ and pick those whose centers lie in $\Omega$. The vortex blob function is taken as

$$
\zeta(x)= \begin{cases}\frac{7}{2 \pi}\left(1-10|x|^{3}+15|x|^{4}-6|x|^{5}\right), & |x|<1 \\ 0, & |x| \geq 1\end{cases}
$$

hence

$$
\int_{\mathbb{R}^{2}} \zeta(x) d x=1
$$

The function $\zeta$ satisfies the condition (17). Since it is axisymmetric and compactly supported, the solution of $-\triangle g=\zeta_{\varepsilon}(x)$ on $\mathbb{R}^{2}$ can be expressed explicitly:

$$
g(x)= \begin{cases}-\frac{7}{2 \pi} \log |x|\left(\frac{1}{2} \frac{|x|^{2}}{\varepsilon^{2}}-2 \frac{|x|^{5}}{\varepsilon^{5}}+\frac{5}{2} \frac{|x|^{6}}{\varepsilon^{6}}-\frac{6}{7} \frac{|x|^{7}}{\varepsilon^{7}}\right), & |x|<\varepsilon, \\ -\frac{1}{2 \pi} \log |x|, & |x| \geq \varepsilon,\end{cases}
$$

which simplifies our computation. The boundary value problem $(10),(11)$ is solved by the Fourier method. First we employ a linear combination of $g$ 's to get a particular solution to the nonhomogeneous equation, then solve the Laplace equation with nonhomogeneous boundary conditions. Let the vorticity $\omega^{\varepsilon}$ be expressed in terms of (9); then the equations for $X_{j}^{\varepsilon}$ and $\alpha_{j}^{\varepsilon}$ are (8) and

$$
\frac{d \alpha_{j}^{\varepsilon}}{d t}=F\left(X_{j}^{\varepsilon}(t), t\right) h^{2} .
$$

We use an explicit Euler scheme to solve these ordinary differential equations. To prevent any vortex blob from moving across the boundary, we use the following modification for those vortex blobs neighboring the boundary: Let $(r, \theta)$ be the 


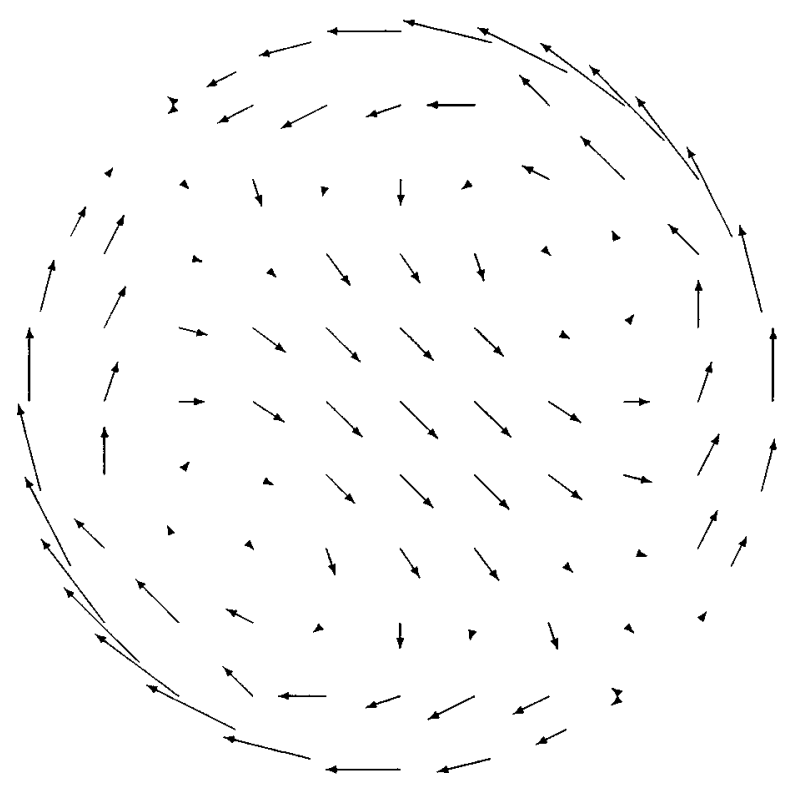

Figure 1

position of a particle and $\left(u_{r}, u_{\theta}\right)$ be its velocity in polar coordinates. Since $u_{r}$ vanishes on the boundary, we solve

$$
\begin{aligned}
\frac{d \bar{r}}{d t} & =u_{r} \frac{1-\bar{r}}{1-r}, \\
\left.\bar{r}\right|_{t=0} & =r
\end{aligned}
$$

which gives

$$
\bar{r}=1-(1-r) e^{-u_{r} t /(1-r)} .
$$

Consequently after a time step $\Delta t$ we take the new coordinate of this particle as $\left(1-(1-r) e^{-u_{r} \Delta t /(1-r)}, \theta+u_{\theta} \Delta t / r\right)$.

The second scheme (B) is the one introduced in [15] and [17]. We extend the domain $\Omega$ a little and evaluate the motion of all vortex blobs even if their centers are not in $\Omega$. The velocity of these blobs is obtained by extrapolation. Since we don't care if the centers are in $\Omega$, the modification for the blobs near the boundary mentioned above is not necessary.

The third scheme $(\mathrm{C})$ is the one developed in this paper. We create overlapped meshes. We consider the contribution of some blobs outside the domain, but extrapolation is not employed. We use the partition of unity here, so we define a compactly supported function

$$
\varphi(x)= \begin{cases}1, & |x|<1-2 \delta \\ \frac{2}{\delta^{3}}\left(|x|+\frac{5}{2} \delta-1\right)(1-\delta-|x|)^{2}, & 1-2 \delta \leq|x|<1-\delta \\ 0, & |x|>1-\delta .\end{cases}
$$

The flow is shown in Figure 1. The meshes of the schemes (A),(B), and (C) are shown in Figures 2, 3, and 4, respectively. For the sake of clearness we take large parameters $h=0.25, \varepsilon=0.5$ and $\delta=0.2$, and only part of the squares are drawn. 


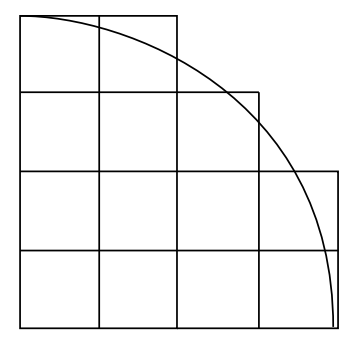

Figure

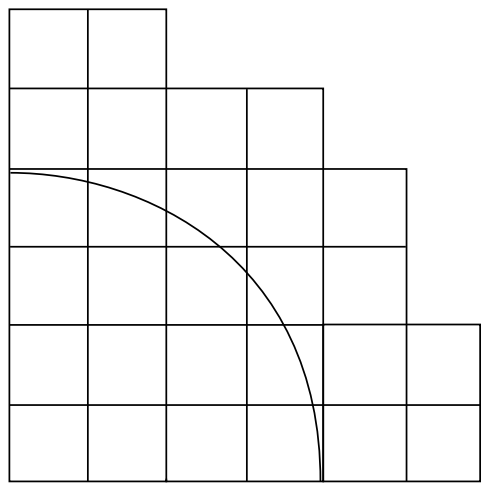

FigURE

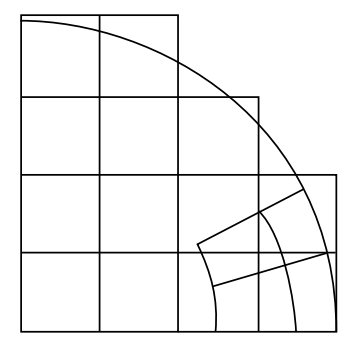

FIGURE

TABLE 1

\begin{tabular}{|c|ccc|}
\hline $\mathrm{t}$ & $(\mathrm{A})$ & $(\mathrm{B})$ & $(\mathrm{C})$ \\
\hline 0 & 0.475 & 0.139 & 0.204 \\
0.1 & 0.486 & 0.152 & 0.182 \\
0.2 & 0.433 & 0.161 & 0.167 \\
0.3 & 0.367 & 0.162 & 0.154 \\
0.4 & 0.373 & 0.155 & 0.142 \\
0.5 & 0.340 & 0.156 & 0.153 \\
\hline 0.6 & 0.304 & 0.152 & 0.169 \\
0.7 & 0.252 & 0.152 & 0.210 \\
0.8 & 0.218 & 0.144 & 0.221 \\
0.9 & 0.183 & 0.139 & 0.254 \\
1.0 & 0.182 & 0.129 & 0.232 \\
\hline 1.1 & 0.162 & 0.132 & 0.218 \\
1.2 & 0.186 & 0.117 & 0.210 \\
1.3 & 0.176 & 0.115 & 0.178 \\
1.4 & 0.162 & 0.105 & 0.175 \\
1.5 & 0.156 & 0.100 & 0.155 \\
\hline 1.6 & 0.142 & 0.0955 & 0.125 \\
1.7 & 0.121 & 0.0901 & 0.112 \\
1.8 & 0.118 & 0.0857 & 0.110 \\
1.9 & 0.109 & 0.0828 & 0.112 \\
2.0 & 0.0976 & 0.0814 & 0.111 \\
\hline
\end{tabular}

In the real computation we take $h=0.1, \varepsilon=0.3, \delta=0.1$ and $\Delta t=0.01$. In the Fourier method we take 100 integral points and we truncate the Fourier series by taking 20 terms. For each scheme we evaluate 200 steps. The errors are shown in Table 1; they are defined as

$$
\text { error }=\max _{j} \max \left\{\left|\left(u_{1}^{\varepsilon}-u_{1}\right)\left(X_{j}^{\varepsilon}(t), t\right)\right|,\left|\left(u_{2}^{\varepsilon}-u_{2}\right)\left(X_{j}^{\varepsilon}(t), t\right)\right|\right\} .
$$

Let us notice that $\left|u_{1}\right|_{\max }=\left|u_{2}\right|_{\max }=1+\sqrt{2}$. 
From the results we may conclude that:

1. To see the consistency we examine the errors at $t=0$, because they are just the truncation errors. The best one is (B) and the worst is (A).

2. All schemes seem stable. Even more, they are asymptotically stable. After a period of oscillation the errors decrease monotonically. We think this is because these schemes are self-adaptive. In particular, the oscillation of the scheme (B) is the smallest.

3. It seems the scheme (B) is the best, but the extrapolation process in it depends on the smoothness of the solution, and it is time-consuming. We use an IBM 486 PC computer. The CPU time for them are 30, 90, and 40 minutes respectively.

4. For the scheme (A) we cannot prove anything theoretically: consistency, stability, or convergence. For all these schemes we don't know how to study the asymptotic stability.

\section{ACKNOWLEDGMENT}

This work was accomplished when the author visited the Research Institute for Mathematical Sciences of Kyoto University as a visiting professor. The author is grateful to the institute and Professor Okamoto for their hospitality. The author also expresses his thanks to the referee who give some valuable comments to help the author revising this manuscript.

\section{REFERENCES}

1. R. A. Adams, Sobolev Spaces, Academic Press, 1975. MR 56:9247

2. S. Agmon, The $L_{p}$ approach to the Dirichlet problems, Anali della Scuola Sup. Pisa, 13, 1959, 405-448. MR 23:A2609

3. S. Agmon A. Douglis and L.Nirenberg, Estimates near the boundary for solutions of elliptic partial differential equations satisfying general boundary conditions I, Comm. Pure Appl. Math., 17, 1959, 623-727. MR 23:A2610

4. C. Anderson and C. Greengard, On vortex methods, SIAM J. Numer. Anal., 22, 1985, 413-440. MR 86j:76016

5. J. T. Beale and A. Majda, Vortex methods I: Convergence in three dimensions, Math. Comp., 39, 1982, 1-27. MR 83i:65069a

6. J. T. Beale and A. Majda, Vortex methods II: Higher accuracy in two and three dimensions, Math. Comp., 39, 1982, 29-52. MR 83i:65069b

7. C. Chiu and R. A. Nicolaides, Convergence of a higher-order vortex method for two dimensional Euler equations, Math. Comp., 51, 1988, 507-534. MR 84c:65117

8. A. J. Chorin, Numerical study of slightly viscous flow, J. Fluid Mech., 57, 1973, 785-796. MR 52:16280

9. O. H. Hald, Convergence of vortex methods for Euler's equations, II, SIAM J. Numer. Anal., 16, 1979, 726-755. MR 81b:76015b

10. O. H. Hald, Convergence of vortex methods for Euler's equations III, SIAM J. Numer. Anal., 24, 1987, 538-582. MR 88i:76003

11. O. H. Hald and V. M. DelPrete, Convergence of vortex methods for Euler's equations, Math. Comp., 32, 1978, 791-809. MR 81b:76015a

12. R. A. Nicolaides, Construction of higher order accurate vortex and particle methods, Appl. Numer. Math., 2, 1986, 313-320. MR 87k:65119

13. P. A. Raviart, An analysis of particle methods, Lecture Notes in Mathematics vol. 1127, Springer-Verlag, 1985, 243-324. MR 87h:76010

14. R. Temam, On the Euler equations of incompressible perfect fluids, J. Funct. Anal., 20, 1975, 32-43. MR 55:3573

15. Lung-An Ying, Convergence of vortex methods for initial boundary value problems, Advances in Math. (China), 20, 1991, 86-102. MR 92b:65103

16. Lung-An Ying, Convergence of vortex methods for three dimensional Euler equations in bounded domains, SIAM J. Numer. Anal., 32, 1995, 542-559. MR 96a:76082 
17. Lung-An Ying and P. Zhang, Fully discrete convergence estimates for vortex methods in bounded domains, SIAM J. Numer. Anal., 31, 1994, 344-361. MR 95b:65131

18. Lung-An Ying and P. Zhang, Vortex Methods, Science Press, Bejing, 1997.

Department of Mathematics, Peking University

Research Institute for Mathematical Sciences, Kyoto University

Current address: School of Mathematical Sciences, Peking University, Beijing, 100871, China

E-mail address: yingla@sxx0.math.pku.edu.cn 